\title{
CAPACIDADE PARA O TRABALHO E RISCO CARDIOVASCULAR EM TRABALHADORES DA PREFEITURA DE UM CAMPUS UNIVERSITÁRIO*
}

Aline Loiola Moura', Maria do Carmo Lourenço Haddad², José Carlos Dalmas³, Vanda Elisa Andres Felli Paloma de Souza Cavalcante Pissinati ${ }^{5}$

'Enfermeira. Mestre em Enfermagem. Secretaria Municipal de Saúde de Apucarana. Apucarana-PR-Brasil.

${ }^{2}$ Enfermeira. Doutora em Enfermagem. Docente da Universidade Estadual de Londrina. Londrina-PR-Brasil.

${ }^{3}$ Matemático. Doutor em Engenharia de Produção. Docente da Universidade Estadual de Londrina. Londrina-PR-Brasil.

${ }^{4}$ Enfermeira. Doutora em Enfermagem. Docente da Escola de Enfermagem da Universidade de São Paulo EEUSP. São Paulo-SP-Brasil. ${ }^{5}$ Enfermeira. Mestranda em Enfermagem da Universidade Estadual de Londrina. Londrina-PR-Brasil.

RESUMO: Este estudo objetivou verificar a relação do Índice de Capacidade para o Trabalho com o Risco Cardiovascular em trabalhadores da prefeitura de um campus universitário. Trata-se de uma pesquisa descritiva, exploratória, transversal e quantitativa realizada em $2012 \mathrm{com}$ análise de prontuário de 226 trabalhadores submetidos a exames periódicos. O risco cardiovascular foi calculado por meio do Escore de Risco de Framingham. Observou-se que 85,0\% dos trabalhadores possuíam mais de 40 anos de idade. Quanto à capacidade para o trabalho, 42,5\% apresentaram capacidade inadequada. Identificou-se que 31,2\% dos trabalhadores apresentaram escore de risco entre médio $(20,8 \%)$ a alto $(10,4 \%)$ para desenvolvimento de doenças cardiovasculares em 10 anos. Conclui-se que apesar de não ter ocorrido associação significativa $(p<0,05)$ entre os trabalhadores com inadequada capacidade para o trabalho e aqueles que apresentaram risco cardiovascular médio e alto houve elevada quantidade de trabalhadores com fatores de risco cardiovasculares.

DESCRITORES: Fatores de risco; Saúde do trabalhador; Avaliação da capacidade de trabalho; Enfermagem.

\section{CAPACITY FOR WORK AND CARDIOVASCULAR RISK AMONG PREFECTURAL WORKERS ON A UNIVERSITY CAMPUS}

\begin{abstract}
This study aimed to ascertain the relationship of the Work Ability Index with Cardiovascular Risk in workers of the prefecture on a university campus. It is descriptive, exploratory, transversal and quantitative research undertaken in 2012 through the analysis of medical records of 226 workers who underwent regular examinations. Cardiovascular risk was calculated using the Framingham Risk Score. It was observed that $85.0 \%$ of the workers were aged over 40 years old. Regarding work capacity, $42.5 \%$ presented inadequate capacity. It was identified that $31.2 \%$ of the workers had a risk score between medium (20.8\%) and high (10.4\%) for developing cardiovascular diseases within 10 years. It is concluded that in spite of there being no significant association $(p<0.05)$ between the workers with inadequate capacity for work and those who presented medium and high cardiovascular risk, there was a high number of workers with cardiovascular risk factors.
\end{abstract}

DESCRIPTORS: Risk factors; Worker's health; Evaluation of work capacity; Nursing.

\section{CAPACIDAD PARA EL TRABAJOY RIESGO CARDIOVASCULAR EN TRABAJADORES DE AYUNTAMIENTO DE UN CAMPUS UNIVERSITARIO}

RESUMEN: Este estudio tuvo la finalidad de verificar la relación del Índice de Capacidad para el Trabajo con Riesgo Cardiovascular en trabajadores del ayuntamiento de un campus universitario. Es una investigación descriptiva, exploratoria, transversal y cuantitativa realizada en 2012 con análisis de prontuario de 226 trabajadores sometidos a exámenes periódicos. El riesgo cardiovascular fue calculado por medio del Score de Riesgo de Framingham. Se observó que $85,0 \%$ de los trabajadores tenían más de 40 años de edad. Cuanto a la capacidad para el trabajo, $42,5 \%$ presentaron capacidad inadecuada. Se identificó que $31,2 \%$ de los trabajadores presentaron score de riesgo entre medio $(20,8 \%)$ y alto $(10,4 \%)$ para desarrollo de enfermedades cardiovasculares en 10 años. Se concluye que a pesar de no haber ocurrido asociación significativa $(p<0,05)$ entre los trabajadores con inadecuada capacidad para el trabajo y aquellos que presentaron riesgo cardiovascular medio y alto, hubo elevada cantidad de trabajadores con factores de riesgo cardiovasculares.

DESCRIPTORES: Factores de riesgo; Salud del trabajador; Evaluación de la capacidad de trabajo; Enfermería.

*Artigo extraído da Dissertação intitulada: Capacidade para o trabalho e risco para doenças cardiovasculares em trabalhadores da prefeitura de um campus universitário público. Universidade Estadual de Londrina, 2013.

Autor Correspondente:

Paloma de Souza Cavalcante Pissinati

Universidade Estadual de Londrina

Av. Robert Koch, 60 - 86038-440 - Londrina-PR-Brasil

E-mail: cavalcanteps7@gmail.com
Recebido: $26 / 09 / 2014$ Finalizado: 04/12/2014 


\section{INTRODUÇÃO}

O aumento da população idosa no mercado de trabalho intensificou a preocupação com a saúde e segurança laboral, sobretudo, no que ser refere ao envelhecimento funcional dos indivíduos. A capacidade para o trabalho tornou-se um indicador importante, por avaliar amplamente as condições de saúde física, bem-estar psicossocial, competência individual, condições e organização do trabalho(1).

A capacidade para o trabalho compreende o nível de aptidão que o trabalhador possui para executar suas atividades laborais diárias, de acordo com as exigências do trabalho, condições físicas e mentais, bem como seu estado de saúde ${ }^{(2)}$. $\mathrm{O}$ modelo finlandês proposto para a manutenção desta capacidade pressupõe a necessidade de integração entre diferentes áreas de ação, as quais englobam melhorias das condições de trabalho, melhorias na organização e no ambiente psicossocial do trabalho, promoção da saúde e dos recursos individuais e desenvolvimento da competência profissional(1).

No início da década de 1980 pesquisadores finlandeses desenvolveram o Índice de Capacidade para o Trabalho (ICT), com o objetivo de permitir autoavaliação do trabalhador sobre sua capacidade para o trabalho( ${ }^{(3)}$. Este índice constitui um protocolo avaliativo da saúde e capacidade de trabalho e, dessa forma, pode contribuir para a construção de programas de saúde ocupacional( ${ }^{(4)}$.

No Brasil, os estudos sobre capacidade para o trabalho e envelhecimento funcional iniciaramse após a tradução do questionário do ICT, na década de 90. Nos últimos anos, tornaram-se foco dos pesquisadores da área da saúde, que buscavam compreender as repercussões das questões de transição demográfica e alterações no modo de produção de trabalho para a saúde dos trabalhadores ${ }^{(5)}$.

A versão brasileira do ICT foi validada, considerada satisfatória e confiável para a avaliação da capacidade para o trabalho, após estudo com trabalhadores dos setores de transmissão de energia de uma empresa do setor elétrico da região de Campinas, no estado de São Paulo ${ }^{(6)}$. O instrumento tem como principais vantagens a rápido preenchimento, baixo custo, além de possibilitar a utilização no nível individual e coletivo, de forma a identificar precocemente diminuição na capacidade laboral e subsidiar ações preventivas ${ }^{(1)}$.

Ressalta-se que é relevante priorizar a saúde e a capacidade para o trabalho em todas as fases ativas do trabalhador. Dentre as estratégias utilizadas estão medidas que envolvam o enfermeiro e a equipe interdisciplinar na implantação de programas de manutenção da saúde e prevenção de doenças relacionadas ou não ao trabalho.

Pesquisa(7) $^{(7)}$ em uma prefeitura de um campus universitário, instituição onde esse estudo foi realizado, identificou o perfil sociodemográfico e ocupacional dos trabalhadores e demonstrou envelhecimento marcante nesta população. Além disso, significativa parcela destes profissionais atuava em cargos que poderiam comprometer sua capacidade para o trabalho e desencadear o envelhecimento funcional.

Ao avaliar a capacidade para o trabalho verificou-se que a população do estudo acima mencionado era, predominantemente, masculina, e constatou-se que $41,0 \%$ da população apresentaram capacidade para o trabalho moderada e baixa, ou seja, inadequada. Ainda, muitos exerciam em suas atividades diárias $\mathrm{O}$ levantamento de pesos, permaneciam longas jornadas em pé, com exposição a ruídos, que associados ao estresse mental podem, ao longo dos anos, contribuir para o comprometimento da saúde dos trabalhadores ${ }^{(7)}$.

A saúde representa o fator que exerce maior força sobre a capacidade de trabalho, em específico, quanto à capacidade funcional e presença de doenças ${ }^{(1)}$. Dentre as patologias que podem acometer o trabalhador, as doenças cardiovasculares destacam-se por serem responsáveis pelo maior número de óbitos na população mundial e, atualmente, constituem um grave problema de saúde pública, com implicações nas condições de saúde e de vida da população mundial(8).

Com o aumento da expectativa de vida, observa-se maior ocorrência de doenças crônico-degenerativas, sobretudo, as doenças cardiovasculares $^{(9)}$. Ainda, estas patologias requerem atenção, sobretudo, porque a mortalidade prematura de adultos e as incapacidades, parciais ou completas, repercutem não somente na qualidade de vida e na rede social dos indivíduos acometidos, mas também 
no sistema de saúde(10).

Quantificar o risco cardiovascular, portanto, em uma população é essencial na abordagem deste problema de saúde pública ${ }^{(9)}$. As doenças cardiovasculares podem ser desencadeadas por variáveis genéticas, ambientais, hábitos de vida, os quais são denominados fatores de risco.

Estes fatores são classificados em modificáveis, quando relacionados ao estilo de vida do indivíduo, como tabagismo, etilismo e outros ${ }^{(10)}$; e não modificáveis que incluem o sexo, a idade e a hereditariedade. Neste estudo, considerando a representatividade dos fatores, serão abordados sexo, idade, dislipidemia, tabagismo, hipertensão arterial e diabetes mellitus.

As doenças cardiovasculares podem interferir na capacidade para o trabalho e têm sido objeto de estudos nacionais e internacionais ${ }^{(1-7,11)}$, demonstrando o interesse dos pesquisadores em identificar situações que comprometem a saúde no ambiente laboral e buscar estratégias de prevenção e controle das mesmas.

Assim, este estudo torna-se relevante ao investigar a relação entre a capacidade para o trabalho e o risco de doenças cardiovasculares em trabalhadores de uma universidade.

Considerando as informações acima mencionadas, o presente estudo teve como objetivo verificar a relação do índice de capacidade para o trabalho com o risco cardiovascular em trabalhadores da prefeitura de um campus universitário.

\section{METODOLOGIA}

Trata-se de um estudo descritivo, exploratório, transversal de abordagem quantitativa. A pesquisa foi desenvolvida na prefeitura do campus universitário de uma universidade estadual do norte do Paraná. A população do estudo foi constituída por 226 prontuários de trabalhadores que atuavam na prefeitura do campus da universidade, que realizaram o exame periódico em 2010. Utilizou-se como critério de inclusão, trabalhadores que participaram da pesquisa no mesmo ano, na qual foi avaliado o índice de capacidade para o trabalho.

Os dados foram coletados dos prontuários arquivados no Serviço Especializado em Engenharia de Segurança e em Medicina do Trabalho (SESMT) da instituição onde o estudo foi realizado.
Utilizou-se um instrumento composto por dados referentes ao trabalhador, como idade, função exercida, resultados dos exames laboratoriais realizados em 2010, diagnósticos clínicos, terapêutica e encaminhamentos para especialidades.

Para identificar os riscos para o desenvolvimento de doenças cardiovasculares foi aplicado o Escore de Risco Framingham (ERF), que calcula a probabilidade de desenvolvimento de um evento coronariano em dez anos ${ }^{(12)}$.

Para tanto o ERF utiliza os seguintes parâmetros: sexo, idade, colesterol total, colesterol-HDL, tabagismo, diagnóstico de hipertensão arterial (HA) e diabetes mellitus (DM). A partir da pontuação os escores de risco para doenças cardiovasculares foram classificados em: baixo $(<10 \%)$, médio $(\geq 10 \%$ a $20 \%)$ e alto risco $(>20 \%)^{(2)}$. De acordo com a Sociedade Brasileira de Cardiologia ${ }^{(13)}$, portadores de diabetes mellitus são categorizados como de alto risco para doenças cardiovasculares, independentemente da pontuação do escore.

A coleta de dados dos prontuários ocorreu de novembro de 2011 a março de 2012, obtendo $100 \%$ de prontuários coletados. Após análise dos prontuários e aplicação ERF os dados foram correlacionados com o Índice de Capacidade para o Trabalho (ICT) que foi identificado em estudo realizado em 2010 com os servidores que constituíram a população desta pesquisa ${ }^{(7)}$.

Os dados coletados foram digitados e armazenados em banco de dados no formato Statistical Package of Social Science (SPSS) versão 20 e posteriormente analisados com técnica estatística analítica univariada e bivariada, com aplicação de teste Qui-Quadrado $(p<0,05)$.

Foram atendidos os pressupostos éticos da Resolução do Conselho Nacional de Saúde $196 / 1996^{(14)}$ que regulamenta as pesquisas que envolvem seres humanos, obtendo a aprovação do Comitê de Ética em Pesquisa de uma Universidade Estadual Pública, conforme Certificado de Apresentação para Apreciação Ética (CAAE) $n^{\circ}$ 0233026800011, em 26 de setembro de 2011.

\section{RESULTADOS}

Dos 226 prontuários analisados, constatouse que $13,3 \%$ dos trabalhadores eram do sexo feminino e $86,7 \%$ do sexo masculino. Quanto à 
faixa etária observou-se que a idade variou de 21 a 70 anos, sendo que a média das idades foi de 48,9 anos com desvio padrão de 10,0 anos, o que significa que o grupo possui uma homogeneidade média no que se refere à idade.

Considerandoas características sociodemográficas, a Tabela 1 apresenta a população de estudo segundo, sexo, situação conjugal, idade e escolaridade.

A Tabela 2 apresenta a caracterização profissional dos trabalhadores relacionada à função/atuação, turno, tempo de trabalho na instituição, se possui outro vínculo empregatício, realização de horas extraordinárias e a presença de trabalhadores que se encontram em situação de readequação funcional, exercendo atividades compatíveis com suas limitações físicas ou psíquicas reconhecidas por perícia médica.

Em relação à capacidade para o trabalho classificou-se como adequada e inadequada, sendo categorizada como adequada capacidade, todos aqueles que alcançaram o escore de boa

Tabela 1 - Características sociodemográficas de trabalhadores da prefeitura de um campus universitário público. Londrina, PR, 2012

\begin{tabular}{lcc}
\hline $\begin{array}{l}\text { Características } \\
\text { sociodemográficas }\end{array}$ & $\mathbf{N}=\mathbf{2 2 6}$ & $\mathbf{\%}$ \\
\hline Sexo & 196 & 86,7 \\
\hline Masculino & 30 & 13,3 \\
\hline Feminino & & \\
\hline Idade & 34 & 15 \\
\hline < Ou igual 40 anos & 192 & 85 \\
\hline$>40$ Anos & & \\
\hline Escolaridade & 26 & 11,6 \\
\hline Fundamental incompleto & 40 & 17,6 \\
\hline Fundamental & 131 & 57,9 \\
\hline Médio & 29 & 12,9 \\
\hline Superior & 226 & 100 \\
\hline Total & & \\
\hline
\end{tabular}

capacidade, e inadequada, os que alcançaram escores de moderada e baixa capacidade. Dentre os participantes, 130 (57,5\%) apresentaram capacidade para o trabalho adequada, enquanto 96 $(42,5 \%)$ trabalhadores apresentaram capacidade para o trabalho inadequada. Destaca-se que nenhum dos trabalhadores alcançou o escore de ótima capacidade para o trabalho.

A Tabela 3 apresentaa relaçãoentreoEscoredeRisco Framingham e o Índice de Capacidade para o Trabalho.

Destaca-se que, no presente estudo, não houve associação significativa $(p=0,965)$ entre o risco cardiovascular e a capacidade para o trabalho. Entretanto, os resultados apontam uma elevada quantidade de trabalhadores com inadequada capacidade para o trabalho e risco para desenvolver doenças cardiovasculares.

Tabela 2 - Categoria profissional de trabalhadores da prefeitura de um campus universitário público. Londrina, PR, 2012

\begin{tabular}{lcc}
\hline Características profissionais & $\mathbf{N}=\mathbf{2 2 6}$ & $\mathbf{\%}$ \\
\hline Atuação & & \\
\hline Auxiliar operacional & 197 & 87,4 \\
\hline Técnico & 21 & 9,1 \\
\hline Superior & 08 & 3,5 \\
\hline Tempo de trabalho na instituição & & \\
\hline < 10 Anos & 39 & 17,3 \\
\hline >Ou = 10 anos & 187 & 82,7 \\
\hline Possui outro emprego & & \\
\hline Sim & 12 & 5,3 \\
\hline Não & 214 & 94,7 \\
\hline Realiza hora extra & \\
\hline Sim & 176 & 77,9 \\
\hline Não & 50 & 22,1 \\
\hline Readequado & & \\
\hline Sim & 11 & 5 \\
\hline Não & 215 & 95 \\
\hline Total & 226 & 100 \\
\hline
\end{tabular}

Tabela 3 - Relação entre risco cardiovascular e índice de capacidade para o trabalho de trabalhadores da prefeitura de um campus universitário público. Londrina, PR, 2012

\begin{tabular}{|c|c|c|c|c|c|}
\hline \multirow{3}{*}{$\begin{array}{l}\text { Escore de Risco } \\
\text { Framingham }\end{array}$} & \multicolumn{4}{|c|}{ Índice de Capacidade para o Trabalho } & \multirow{3}{*}{ Valor de $p$} \\
\hline & \multicolumn{2}{|c|}{ Adequada } & \multicolumn{2}{|c|}{ Inadequada } & \\
\hline & $\mathrm{N}$ & $\%$ & $\mathrm{~N}$ & $\%$ & \\
\hline Baixo Risco & 88 & 67,7 & 66 & 68,8 & \\
\hline Médio Risco & 27 & 20,8 & 20 & 20,8 & 0,965 \\
\hline Alto Risco & 15 & 11,5 & 10 & 10,4 & \\
\hline Total & 130 & 100 & 96 & 100 & \\
\hline
\end{tabular}




\section{DISCUSSÃO}

O predomínio da população masculina se assemelha com estudo sobre confiabilidade do ICT em trabalhadores do sul do Brasil, no qual $83 \%$ dos participantes eram do sexo masculino ${ }^{(3)}$. Estudo $^{(2)}$ demonstra que os homens manifestam melhor capacidade para o trabalho quando comparados às mulheres.

Em contrapartida, quando verificado o risco para o desenvolvimento de doenças cardiovasculares, o homem apresenta probabilidade $71 \%$ maior de apresentar três ou mais fatores de risco cardiovasculares do que as mulheres. Resultado que se deve aos hábitos de vida, tais como consumo habitual de gordura, tabagismo, entre outros ${ }^{(15)}$.

Quanto à faixa etária, 85\% possuíam mais de 40 anos de idade, resultado semelhante ao encontrado entre trabalhadores de higiene e limpeza de um hospital universitário ${ }^{(16)}$. O fato dos trabalhadores estarem acima dos quarenta anos, pode representar maior propensão aos fatores de risco cardiovasculares ${ }^{(17)}$ e à diminuição de sua capacidade para o trabalho ${ }^{(18)}$.

Ao analisar o grau de escolaridade da população, constatou-se que a maioria, 57,9\%, concluiu o ensino médio. Resultado semelhante foi encontrado em estudo sobre fatores associados à capacidade para o trabalho de trabalhadores metalúrgicos, fato que qualifica o trabalhador e subsidia no seu autocuidado a fim melhorar ou manter sua capacidade funcional(3).

Em relação à aérea de atuação dos trabalhadores, observou-se que 87,4\% exerciam função de auxiliar operacional. A quantidade expressiva de trabalhadores atuantes em cargos que podem desenvolver atividades consideradas pesadas indica provável diminuição precoce da capacidade para o trabalho.

Ressalta-se que quanto menor o nível do cargo ocupado estima-se menores rendimentos mensais, influenciando assim na qualidade de vida e na saúde do trabalhador que preconiza a alimentação adequada, boas condições de moradia, acesso a serviços de saúde, atividade física regular, entre outros ${ }^{(19)}$.

Entretanto, salienta-se que $42,5 \%$ do total da população apresentaram ICT inadequado. Ao considerar que se trata de uma população com predomínio de idade acima de 40 anos, este resultado pode estar associado ao envelhecimento, o qual desencadeia diversas doenças que favorecem a deterioração da capacidade física e mental e, consequentemente, diminui a capacidade para o trabalho(20).

Ao calcular o risco para doenças cardiovasculares $31,2 \%$ apresentaram risco médio e alto para um evento cardiovascular em 10 anos. Estes dados são superiores ao estudo realizado com profissionais da área da saúde, no qual o médio e alto risco prevaleceu em $11,6 \%{ }^{(21)}$. Resultado alarmante ao constatar percentual significativo de ICT inadequado neste estudo.

Ao analisar a relação entre risco cardiovascular e ICT dos trabalhadores verificou-se que não houve correlação estatisticamente significativa $(p<0,05)$ entre os que apresentaram capacidade para o trabalho inadequada com os que apresentaram risco cardiovascular médio e alto. Apesar disto, a investigação entre a relação da capacidade para o trabalho e o risco cardiovascular faz-se necessária, como evidenciado em estudo ${ }^{(22)}$ realizado com trabalhadores rurais, no qual $1,9 \%$ dos participantes que apresentaram ICT baixo e moderado referiram possuir doenças cardiovasculares.

Diante das repercussões das doenças cardiovasculares para a saúde do trabalhador ${ }^{(8-10)}$, destaca-se a importância da atuação da equipe de enfermagem e, sobretudo, do enfermeiro como profissional capacitado para promover a educação em saúde em todos os níveis de assistência ${ }^{(23)}$ e promover a manutenção da capacidade para o trabalho, monitorando o estado de saúde de forma a adequar as atividades laborais exercidas pelo trabalhador.

\section{CONSIDERAÇÕES FINAIS}

Os resultados do estudo evidenciaram o comprometimento da capacidade para o trabalho dentre a população estudada, visto que $42,5 \%$ apresentaram ICT inadequado. O médio e alto risco para o desenvolvimento de doenças cardiovasculares esteve presente de forma semelhante entre trabalhadores com ICT adequado e inadequado, não houve relação estatisticamente significativa entre estas variáveis.

Entretanto, a elevada quantidade de 
trabalhadores com inadequada capacidade para o trabalho e risco para desenvolvimento de um evento cardiovascular em 10 anos entre médio e alto, reforça a importância de realizar novos estudos, longitudinais que acompanhem os trabalhadores a fim de verificar o comportamento das variáveis em questão.

Os resultados apresentados sugerem a relevância do desenvolvimento de programas de saúde ocupacional, coordenados por enfermeiros, a fim de preservar, restaurar e melhorar a capacidade para o trabalho. Estratégia necessária, visto que as doenças cardiovasculares constituem causas de afastamentos, invalidez e até óbitos prematuros dos trabalhadores.

\section{REFERENCIAS}

1. Martinez MC, Latorre MRDO, Fischer FM. Capacidade para o trabalho: revisão de literatura. Cienc. saude colet. [Internet] 2010;15(Suppl 1). [acesso em 20 nov 2014]. Disponível: http://dx.doi.org/10.1590/S141381232010000700067

2. Tuomi K, Ilmarinen J, Jahkola A, Katajarinne L, Tulkki A. Índice de capacidade para o trabalho. São Carlos: Edufscar, 2005.

3. Renosto A, Biz P, Hennington EA, Pattussi MP. Confiabilidade teste-reteste do índice de capacidade para o trabalho em trabalhadores metalúrgicos do sul do Brasil. Rev. Bras. Epidemiol. [Internet] 2009;12(2). [acesso em 20 nov 2014]. Disponível: http://dx.doi. org/10.1590/S1415-790X2009000200011

4. Moreira PSV, Silvino ZR, Cortez EA. Aplicação do índice de capacidade para o trabalho na enfermagem: estudo descritivo. Online Braz J Nurs. [Internet] 2013;12(Suppl) [acesso em 20 nov 2014]. Disponível: http://www.objnursing.uff.br/index.php/nursing/ article/viewFile/4223/2514

5. Marinho TB, Costa LB, Costa LCA, Alcantara PGF, Santos RLS. Reflexões sobre a capacidade para o trabalho dos professores das escolas municipais de João Pessoa. XXI Encontro nacional de engenharia de produção. [Internet] 2011. [acesso em 20 nov 2014]. Disponível: http://www.abepro.org.br/ biblioteca/enegep2011_tn_wic_138_874_19012. pdf

6. Martinez MC, Latorre MRDO, Fischer FM. Validade e confiabilidade da versão brasileira do índice de capacidade para o trabalho. Rev. Saúde Públ. [Internet]2009;43(3). [acesso em 14 abr 2014]. Disponível: http://dx.doi.org/10.1590/S003489102009005000017
7. Moura AL, Reis LM, Vannuchi MTO, Haddad MCL, Domansky RC. Capacidade para o trabalho de funcionários da prefeitura de um campus universitário público. REE. [Internet] 2013;15(1) [acesso em 14 abr 2014]. Disponível: http://dx.doi.org/10.5216/ree. v15i1.13574

8. Christofaro DGD, Andrade SM, Fernandes RA, Ohara D, Dias DF, Junior IFF, et. al. Prevalência de fatores de risco para doenças cardiovasculares entre escolares em Londrina-PR: diferenças entre classes econômicas. Rev. Bras. Epidemiol. [Internet] 2011;14(1). [acesso em 20 nov 2014]. Disponível: http://dx.doi.org/10.1590/ S1415-790X2011000100003

9. Martins LN, Souza LS, Silva CF, Machado RS, Silva CEF, Vilagra MM, et. al. Prevalência dos fatores de risco cardiovascular em adultos admitidos na unidade de dor torácica de Vassouras, RJ. Rev. Bras. Cardiol. [Internet] 2011;24(5). [acesso em 20 nov 2014]. Disponível:http:// sociedades.cardiol.br/socerj/revista/2011_05/2a_2011_ v24_n05_04prevalencia.pdf

10. Pereira JC, Barreto SM, Passos VMA. Perfil de risco cardiovascular e autoavaliação da saúde no Brasil: estudo de base populacional. Rev. panam. salud publica. [Internet] 2009;25 (6). [acesso em $14 \mathrm{abr}$ 2014]. Disponível: http://dx.doi.org/10.1590/S102049892009000600004

11. Bonsdorff MB, Seitsamo J, Ilmarien J, Nygard $\mathrm{CH}$, Bonsdorff ME, Rantanen T. Work ability as a determinant of old age disability severity: evidence from the 28-year finish longitudinal study on municipal employees. Aging Clin Exp Res. [Internet] 2011;24(4). [acesso em 21 nov 2014]. Disponível: https://jyx. jyu.fi/dspace/bitstream/handle/123456789/40589/ AGING_2012_v24_354s.pdf?sequence=1

12. Galvão NI, Vilela RFJTJ, Orlandi BMM, Ferraz RF, Costa FAA, Fagundes DJ. Determinação do risco cardiovascular em população de check-up espontâneo através do Escore de Framingham. Rev. Bras. Cardiol. [Internet] 2013;26(5) [acesso em 21 nov 2014]. Disponível: http://www.rbconline.org.br/artigo/ determinacao-do-risco-cardiovascular-em-populacaode-check-up-espontaneo-atraves-do-escore-deframingham/

13. Sociedade Brasileira de Cardiologia. I diretriz brasileira de prevenção cardiovascular. Arquivos brasileiros cardiol. [Internet] 2013;101(6) [acesso em 21 nov 2014]. Disponível: http://publicacoes.cardiol. br/consenso/2013/Diretriz_Prevencao_Cardiovascular. pdf

14. Ministério da Saúde (BR). Conselho Nacional de Saúde. Diretrizes e normas regulamentadoras de pesquisa envolvendo seres humanos. Resolução n. 196, de 10 de outubro de 1996. Brasília [Internet]. 1996.Brasil. [acesso em 2014 mar 26]. Disponível: http://conselho. saude.gov.br/docs/Reso196.doc 
15. Muniz LC, Schneider BC, Silva ICM, Matijasevich A, Santos IS. Fatores de risco comportamentais acumulados para doenças cardiovasculares no sul do Brasil. Rev. Saúde Públ. [Internet] 2012;46(3) [acesso em 21 nov 2014. Disponível: http://dx.doi.org/10.1590/ S0034-89102012005000021

16. Silva LG, Haddad MCL, Domansky RC, Vituri DW. Capacidade para o trabalho entre trabalhadores de higiene e limpeza de um hospital universitário público. REE. [Internet] 2010;12(1). [acesso em 14 abr 2014]. Disponível: http://www.revistas.ufg.br/index.php/fen/ article/view/5788/6602

17. Farias RG, Santos SMA. Influência dos determinantes do envelhecimento ativo entre idosos mais idosos. Texto \& contexto enferm. [Internet] 2012;21(1). [acesso em 14 abr 2014]. Disponível:http://dx.doi.org/10.1590/ S0104-07072012000100019

18. Kreling NH. O envelhecimento do trabalhador impõe novos desafios às políticas públicas. Ind. Econ. FEE. [Internet] 2010;38(1) [acesso em 14 abr 2014]. Disponível: http://revistas.fee.tche.br/index.php/ indicadores/article/view/2414/2849

19. Cunha JB, Blank VLG, Boing AF. Tendência temporal de afastamento do trabalho em servidores públicos (19952005). Rev. Bras. Epidemiol. [Internet] 2009;12(2). [acesso em 14 abr 2014]. Disponível: http://dx.doi. org/10.1590/S1415-790X2009000200012

20. Vita A, Palma R, Maganhani CB, Simeão SFAP, Conti MHS, Trize DM, et. al. Nível de capacidade para o trabalho e fatores associados em profissionais de atividades sedentárias. Salusvita. [Internet] 2012;31(3). [acesso em 20 nov 2014]. Disponível em: http://www. usc.br/biblioteca/salusvita/salusvita_v31_n3_2012_ art_07.pdf

21. Cavagioni L, Pierin AMG. Cardiovascular risk among health professionals working in pré-hospital care services. Rev. Esc. Enferm. USP. [Internet] 2012;46(2) [acesso em 20 nov 2014]. Disponível: http://dx.doi. org/10.1590/S0080-62342012000200018

22. Ferreira ESS, Duran ECM, Daniel JGM, Toledo VP. Ability of working among rural workers of sugar and alcohol mill. Rev enferm UFPE. [Internet] 2014;8(2) [acesso em 20 nov 2014]. Disponível: http://www. revista.ufpe.br/revistaenfermagem/index.php/revista/ article/view/3438/pdf_4539

23. Souza SM, Bernardino E, Vicelli RMM, Kalinowski CE. Perfil de pacientes submetidos ao cateterismo cardíaco: subsídios para prevenção de fatores de risco cardiovascular. Cogitare enferm. [Internet] 2014;19(2) [acesso em 26 set 2014]. Disponível: http://ojs.c3sl.ufpr. br/ojs2/index.php/cogitare/article/view/36984/22754 